\title{
An Empirical Analysis of Macroscopic Fundamental Diagrams for Sendai Road Networks
}

\author{
Peng fei $\mathrm{WANG}^{1, *}$, Kentaro WADA ${ }^{2}$, Takashi AKAMATSU ${ }^{1}$ and Yusuke HARA ${ }^{3}$ \\ ${ }^{1}$ Graduate School of Information Sciences, Tohoku University, Sendai 980-8579, Japan \\ ${ }^{2}$ Institute of Industrial Science, The University of Tokyo, Meguro, Tokyo 153-8505, Japan \\ ${ }^{3} \mathrm{New}$ Industry Creation Hatchery Center, Tohoku University, Sendai 980-8579, Japan
}

Received June 2, 2014; final version accepted December 5, 2014

\begin{abstract}
The purpose of this paper is to explore the features of the Macroscopic Fundamental Diagram (MFD) for Sendai road networks. In order to achieve this, we used the observed data of 5-minute periods throughout one year $(5 / 1 /$ 2012-4/30/2013) to draw the MFDs for Sendai road networks. The result of the empirical verification shows the following: (1) the hysteresis loops always form in an MFD on weekdays with good weather, and their formation times correspond to the peak hours of the morning and evening; (2) the shape of the MFD is invariant throughout the year; (3) loops do not form on Sundays (or holidays) when the peak of traffic demand is lower than on weekdays; (4) loops that are larger than those in a typical MFD form when the driving environment is poor; and (5) if the analysis area for drawing the MFD shrinks, then the variation of the MFD expands.
\end{abstract}

KEYWORDS: Macroscopic Fundamental Diagram, traffic congestion, hysteresis loop, detector, Sendai

\section{Introduction}

Traffic congestion remains a significant problem faced by cities worldwide. Various types of sensors (e.g., loop detectors and probe vehicles) are installed in urban road networks to monitor and measure traffic congestion on individual links or road sections. However, it is still difficult to understand and directly evaluate network wide traffic states or network performance based on disaggregated data of individual links. This is because (disaggregated) traffic flow dynamics on congested networks are complex and chaotic owing to interactions between nonlinear traffic flow phenomena and drivers' route choice behaviors.

As an alternative to the disaggregate approach, Daganzo (2007) proposed a macroscopic congestion measure called the Macroscopic Fundamental Diagram (MFD), which captures urban traffic states at an aggregate level. The MFD relates the number of vehicles (or space-mean density) in an area to the area's trip completion rate (or space-mean flow) if the network in the area is homogeneously congested and demand evolves slowly over time. Geroliminis and Daganzo (2008) demonstrated that the well-defined MFD, which is reproducible and invariant when the demand changes both within-day and day-to-day, exists with a field experiment in downtown Yokohama, Japan (see Fig. 1).

A well-defined MFD is a useful measure for establishing a robust traffic policy because we can simply understand the area's traffic state using average density only, and control parameters can be flexibly adjusted based on real time information. For example, we can prevent a road network from attaining a congested state by regulating area inflow rates to maintain an average density that is less than the MFD critical density (for examples, see Daganzo, 2007; Yoshii et al., 2010).

However, some empirical studies have shown that MFDs with high scatters and hysteresis loops exist for some network types, such as heterogeneous networks or freeways (e.g., Buisson and Ladier, 2009; Geroliminis and Sun, 2011; Saberi and Mahmassani, 2012; Saberi and Mahmassani, 2013; Knoop and Hoogendoorn, 2013). Therefore, to use the MFD concept in practice, we need to answer the question "what kind of conditions are needed to observe the welldefined MFD in real world road networks" by conducting additional empirical studies. However, the number of MFD empirical studies is limited. Furthermore, these studies use at most only several days' worth of data, which may be insufficient to investigate the MFD's reproducibility.

We address this deficiency in MFD analyses of real world road networks by exploring MFD features for Sendai road networks with long-term data (5/1/2012-4/30/2013) observed by fixed detectors. We found that a 24-hour MFD in Sendai road networks always forms hysteresis loops on sunny weekdays (we call this the standard condition), and the MFD shape is invariant throughout the entire year. Furthermore, the MFD shape may change with different external

This work is partially supported by the Japan Society for the Promotion of Science, Grant-in-Aid for Young Scientists (B) 26820207.

*Corresponding author. E-mail: wpf@plan.civil.tohoku.ac.jp 


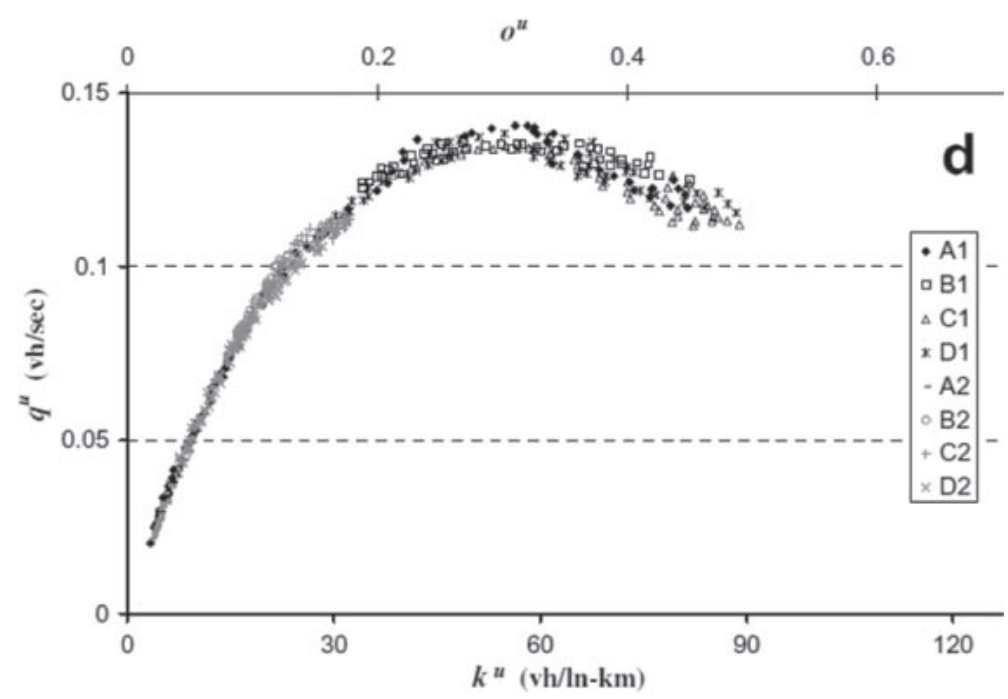

Fig. 1. Well-defined MFD for Yokohama downtown arterial networks (Geroliminis and Daganzo, 2008).

conditions such as traffic demand, weather, and analysis area size. We believe that this makes it necessary to conduct this type of research before applying the MFD concept in practice.

The remainder of this paper is organized as follows: Section 2 describes the Sendai road networks and the data that is directly obtained from fixed detectors. The first half of Section 3 presents the MFD definition and typical MFD features, and the second half presents an analysis of the effect of various external conditions, such as traffic demand, weather, and analysis areas, on MFD shape. Finally, Section 4 presents a discussion of the results and suggests directions for further research.

\section{Observational Data}

We use the Sendai metropolitan area road networks (excluding freeways) for analysis in this study. Sendai is the largest city in the Tohoku region, with a population of about 1.07 million. The road network link has $2-4$ lanes in each direction and the speed limit is about $60 \mathrm{~km} / \mathrm{h}$. The distance between the intersections is $100-300 \mathrm{~m}$, and most intersections are controlled by multiphase traffic signals (signal cycle length may change with the time of day, e.g., $90 \mathrm{~s}$ in the day and $120 \mathrm{~s}$ at night).

We use the traffic flow and traffic density collected by 1,756 detectors ( 878 links) for the analysis (drawing the MFD) (see Fig. 2). The analysis period is one year, from 5/1/2012 to 4/30/2013 (a total of 365 days)*. The detectors record values for each 5-minute period during each 24-hour day, producing a total number of periods as $288(T=288)$ for each day. Detector $i$ observes the traffic flow $q_{t}^{i}$ and vehicle speed $v_{t}^{i}$ in each time period $t$. The traffic density is calculated by $k_{t}^{i}=q_{t}^{i} / v_{t}^{i}$.

\section{MFD Definition and Characterization}

We explore the qualitative features by drawing the daily MFD using the detector data throughout the entire year. First, we provide the definition (and the method for drawing the MFD) for the analysis in Section 3.1. We describe an overview of the MFD features, which are explored from observations throughout the entire year, in Section 3.2. In Section 3.3, we discuss the features of the typical MFD in more detail. Finally, we analyze the effects of traffic demand, weather, and area division on the features of the MFD in Sections 3.4-3.6, respectively.

\subsection{MFD definition}

In this section, we define the MFD and the method for drawing it using an example. Figure 3 indicates that the horizontal axis represents the average flow and the vertical axis represents the average density. Each plotted point is the pair (average density $\bar{k}_{t}$, average flow $\bar{q}_{t}$ ) observed during each time period $t(t=1,2, \ldots, T)$ for each day. The average density $\bar{k}_{t}$ (the horizontal coordinate) is the average value of the total density during time period $t$, which is the sum of $k_{t}^{i}$ observed by the detector $i$ for the entire network:

*However, due to detector failure, some records are missing for 28 days (about 7\%) of the 365 -day period. We omitted these days before we conducted the analysis. 


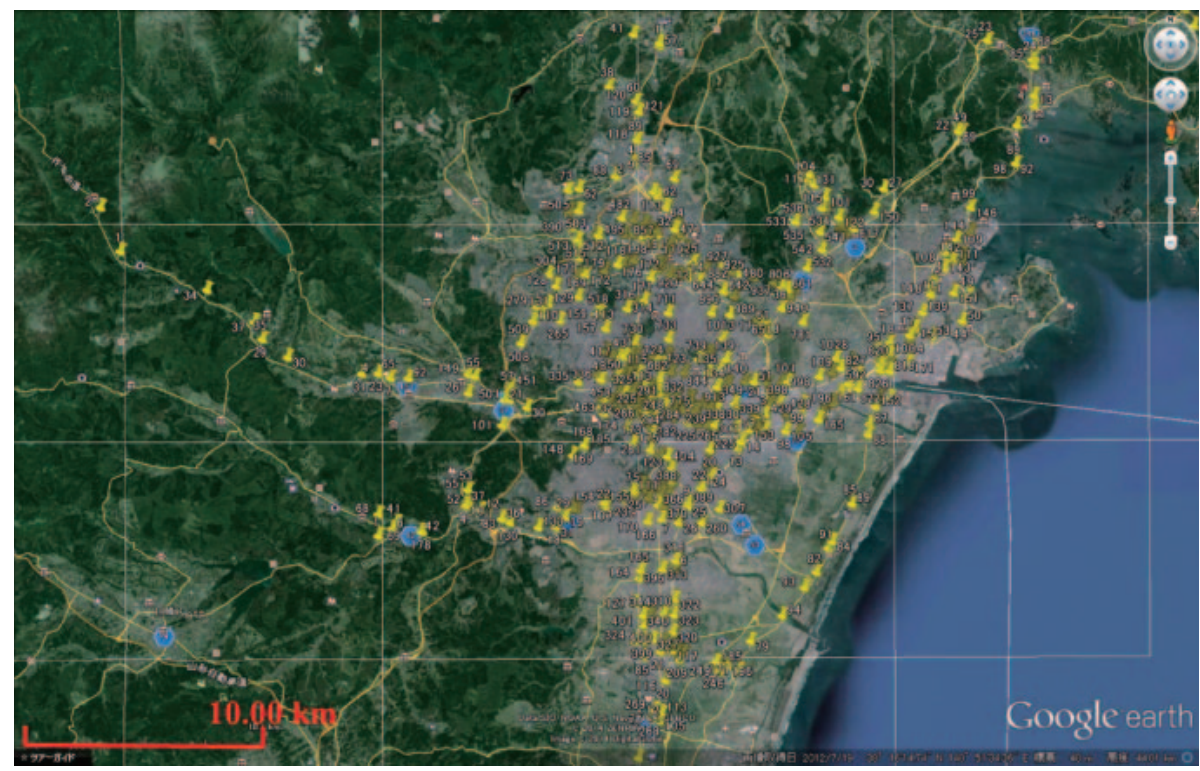

Fig. 2. Locations of all links with detectors in Sendai road networks.

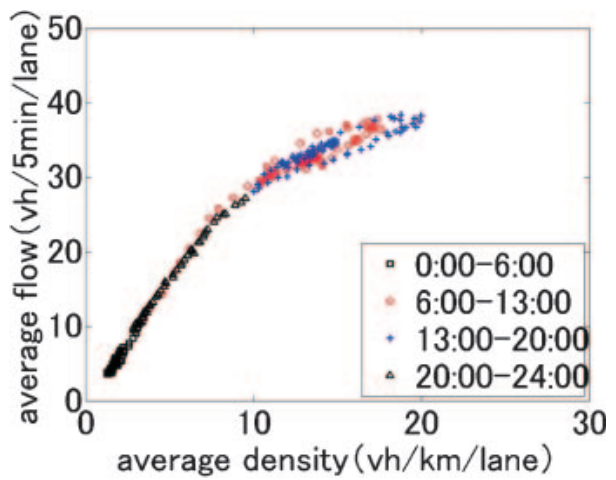

(a) $9 / 14 / 2012$ (Friday, sunny)

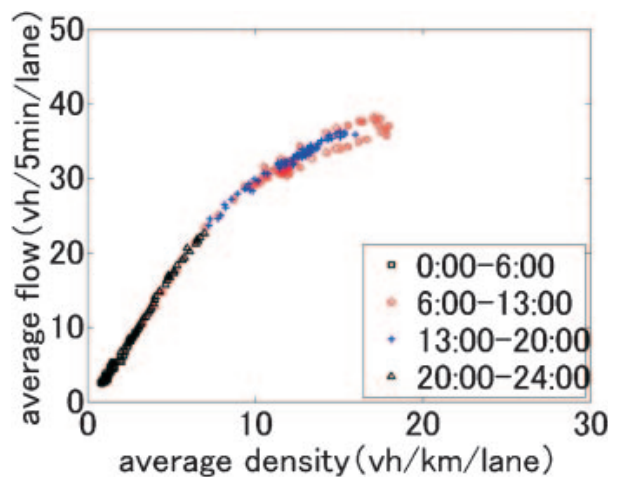

(b) 11/19/2012 (Monday, sunny)

Fig. 3. Typical MFD.

$$
\bar{k}_{t}=\frac{\sum_{i=1}^{I_{0}}\left(q_{t}^{i} / v_{t}^{i}\right)}{\left|I_{0}\right|}
$$

The average flow $\bar{q}_{t}$ (the vertical coordinate) is the average value of the total flow during time period $t$, which is the sum of $q_{t}^{i}$ observed by the detector $i$ for the entire network:

$$
\bar{q}_{t}=\frac{\sum_{i=1}^{I_{0}} q_{t}^{i}}{\left|I_{0}\right|}
$$

where $\left|I_{0}\right|$ in Eqs. (3.1) and (3.2) is the number of detectors in set $I_{0}$. We use the detector set $I_{0}$ (see Fig. 2) in the average operation above and in Sections 3.2-3.5.

In addition, we divide the 24 hours in each day into four time periods (see Fig. 3: 0:00-6:00, 6:00-13:00, 13:0020:00, and 20:00-24:00.). We show each time period with a different mark (for example, the data for 6:00-13:00 is shown with the "red o," and the data for 13:00-20:00 is shown with the "blue +").

\subsection{MFD features and classification}

We drew the daily MFD for the entire year, using detector set $I_{0}$ as the benchmark case. Numerous MFD observations reveal that the daily MFD throughout the entire year can be classified into several types depending on its shape. Among them, one is the "typical MFD" that appears on weekdays in which the weather is good (this is the most common type throughout the year, and we call it the standard condition). All the standard condition MFDs have the following two common features (see Section 3.3): 
- A hysteresis loop always exists.

- The shape of the MFD is invariant throughout the entire year.

However, when we view the MFD in more detail under various conditions, we find that the shape of the MFD could have been changed by any of the following:

(1) the demand condition of the road network,

(2) the supply condition of the road network, or

(3) the area setting in the road networks selected for analysis.

Specifically, for (1), the weekend MFD, in which the time-series of the traffic demand pattern is distinctly different from that of weekdays, a single loop either forms or does not form during one day. For (2), the MFD of a bad weather day (e.g., snow), in which the vehicle driving environment is distinctly different from that of sunny days, a loop forms which is larger than a typical MFD loop. For (3), if the analysis area (the detector set) for drawing the MFD becomes smaller, the MFD variation becomes larger. We provide a detailed analysis of these results in Sections 3.4-3.6, respectively.

\subsection{Typical MFD}

We find that the significant feature of the typical MFD (a weekday with good weather) is the formation of a loop (see, Fig. 3). We express this feature more clearly by dividing the 24-hours of data into two parts, which are the morning (0:00-13:00) and the evening (13:00-24:00) to draw the MFD. From Figs. 4 and 6, we find that

- in the morning, a single loop always forms (see Figs. 4(a) and 6(a));

- in the evening, a loop may form (see, Fig. 4(b)), or it may not (see Fig. 6(b)).

We can explain these typical MFD loops in conjunction with the time-series pattern of the average flow and average density (see Fig. 5). First, Figs. 4(a), 4(b) and Figs. 5(a), 5(b) show that the coordinates of plots of the loop on the MFD correspond to the pair of measurements (average density and average flow) in peak hours. Indeed, the loop forms on the MFD in the morning from 7:30-8:30 and in the evening from 17:30-18:30. Furthermore, as shown in Figs. 5(a), 5(b), we find that during the peak time period, the average flow begins to decrease immediately after time (17:45), when the average flow is at its maximum value (38.4), and the average density remains near its maximum (19.7-19.9) for about one half hour (17:50-18:20). This shows that average density does not decrease greatly near the end of the right side of

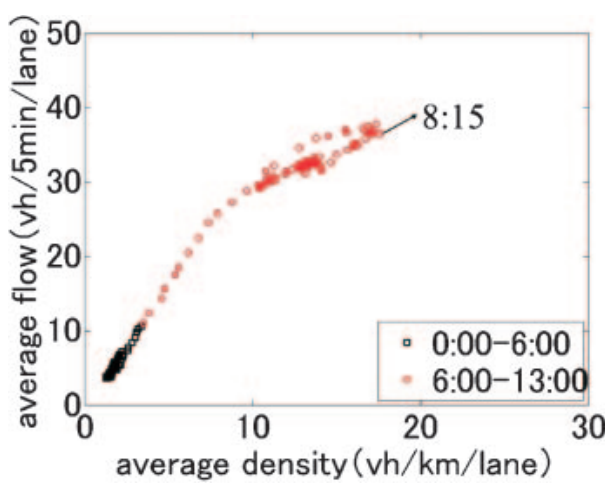

(a) 0:00-13:00

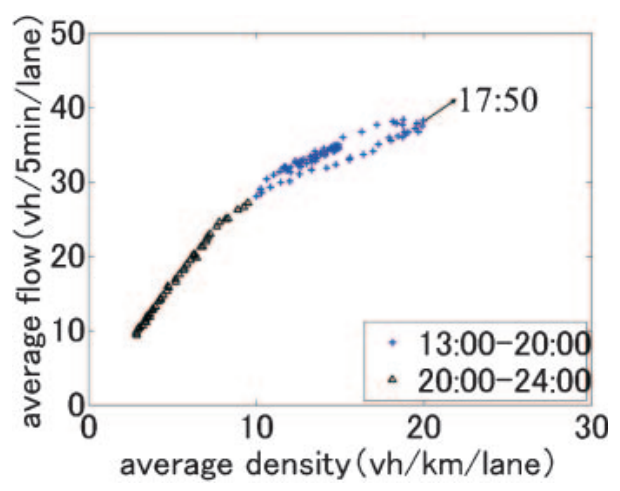

(b) 13:00-24:00

Fig. 4. MFD in the morning and evening of $9 / 14 / 2012$.

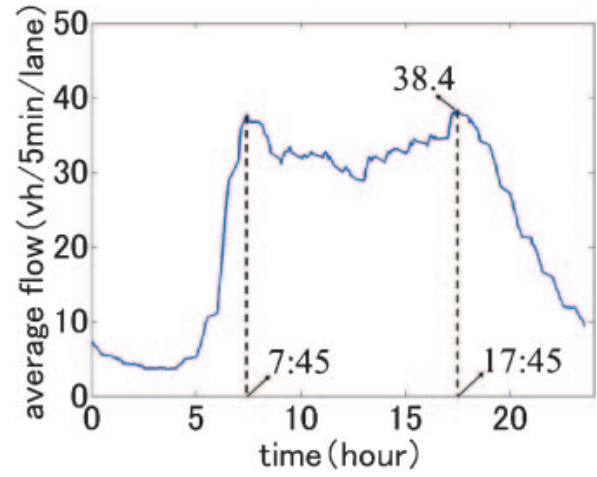

(a) Average flow

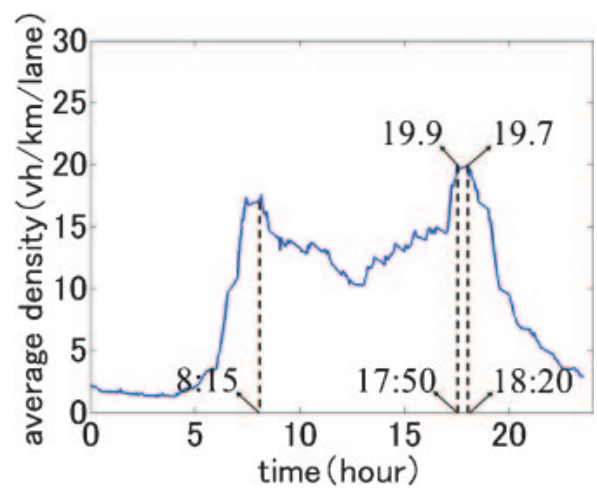

(b) Average density

Fig. 5. Time-series of the average flow and average density of 9/14/2012. 


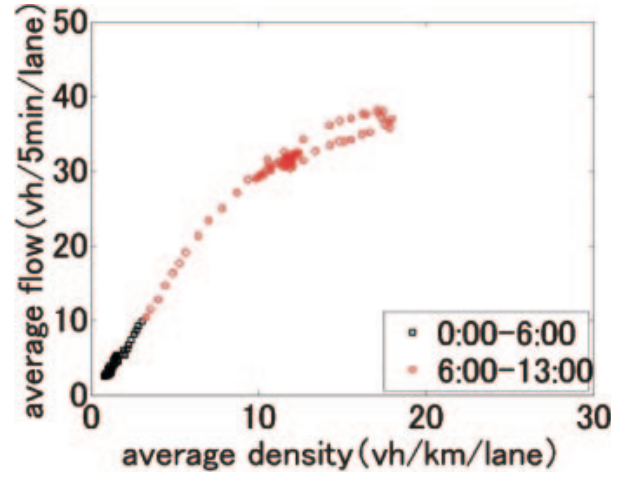

(a) 0:00-13:00

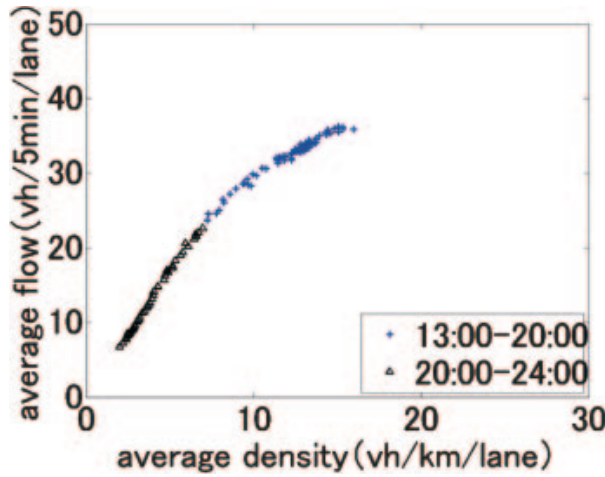

(b) 13:00-24:00

Fig. 6. MFD in the morning and evening of $11 / 19 / 2012$.

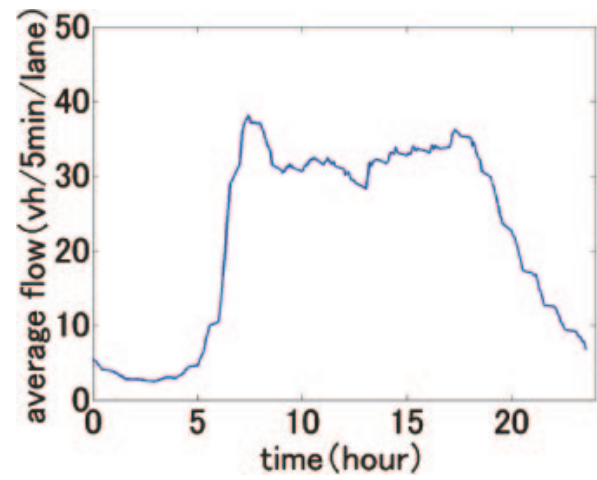

(a) Average flow

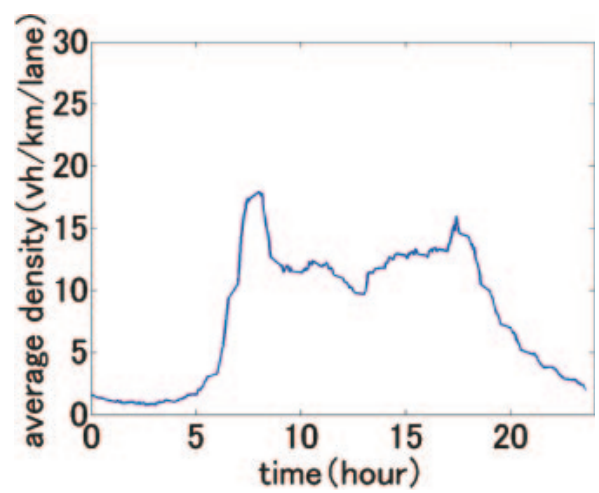

(b) Average density

Fig. 7. Time-series of the average flow and average density of 11/19/2012.

the upper curve of the loop; only the average flow transits to the (lower value than the upper curve) lower curve. This means that for the standard condition, the average density dose not decrease rapidly after the end of the peak of the average flow, and a (hysteresis) loop appears on the $\mathrm{MFD}^{\dagger}$. In the following, we discuss the evening case in which the loop does not form (see, Figs. 3(b) and 7) in Section 3.4.

\subsection{Traffic demand effect}

The MFD shapes and features may possibly change in conjunction with the level of traffic demand and its time-series pattern. Indeed, by observing the MFDs for the weekends throughout the entire year, we find that they all share the following common features:

- A large single loop forms during one day in a Saturday MFD (see Fig. 8(a)).

- The explicit loop seldom appears in a Sunday (or holiday) MFD (see Fig. 9(a)).

Such MFD features on Saturday and Sunday (or a holiday) can be explained in conjunction with the features of their traffic demand. In the following, we analyze the relationship between the time-series pattern of the traffic demand and the MFD in detail. Figure 8(b) shows that the average traffic flow on Saturday does not have a prominent peak in the morning (as is found for standard conditions), it increases very slowly with slope $S_{1}^{f}$ during 7:30-17:25, and then swiftly decreases with slope $S_{2}^{f}$ until 20:00. On the other hand, the average density increases gradually with slope $S_{1}^{d}$ during 13:00-17:15 (see Fig. 8(c)). This increase until 17:15 corresponds to the state transition on the upper curve with slope $S_{1}^{f} / S_{1}^{d}$ of the MFD. The average density then maintains its high values during 17:15-17:35, and begins to decrease with slope $S_{2}^{d}$ at 17:35, while the average traffic flow begins to decrease with slope $S_{2}^{f}$ at 17:25. This decrease corresponds to the state transition on the lower curve with $S_{2}^{f} / S_{2}^{d}$ of the MFD. Because the average flow decreases rapidly with congestion spread, the decreasing slope $S_{2}^{f} / S_{2}^{d}$ of the MFD is larger than the increasing slope $S_{1}^{f} / S_{1}^{d}$ of the MFD. As a result, we observe that the state changes clockwise along the upper curve when increasing and along the lower curve (hysteresis loop) when decreasing. The MFD hysteresis loop does not form on Sundays because the traffic demand is low throughout that day. The average density is also low and has no prominent peak during that day (see Fig. 9).

${ }^{\dagger}$ We estimate that this behavior of average flow and average density corresponds to the spread process of traffic congestion. We will attempt to verify the concrete mechanism in future research. 


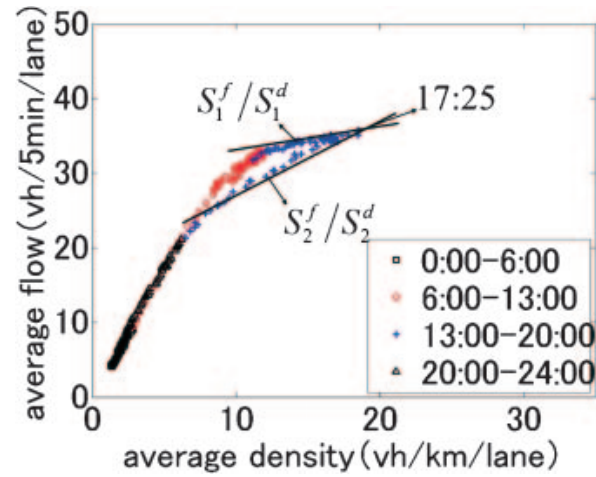

(a) MFD

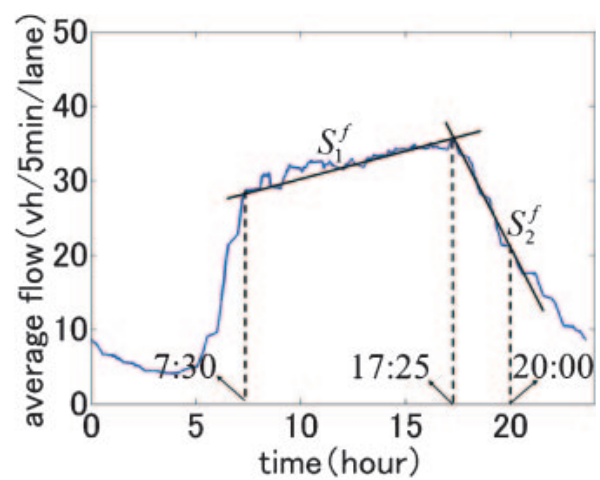

(b) Average flow

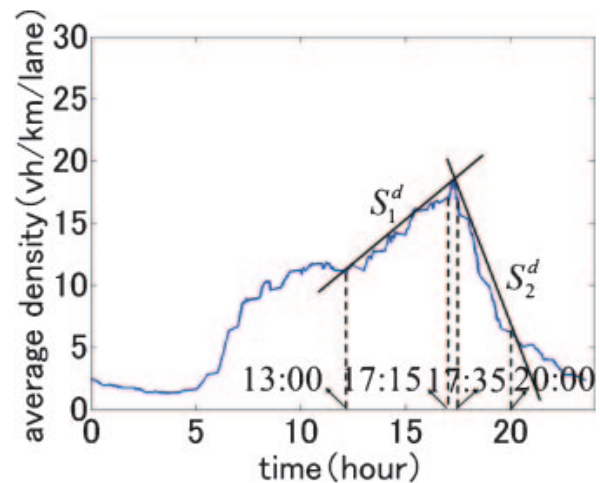

(c) Average density

Fig. 8. MFD, time-series of the average flow, and average density of 11/10/2012 (Saturday, sunny).

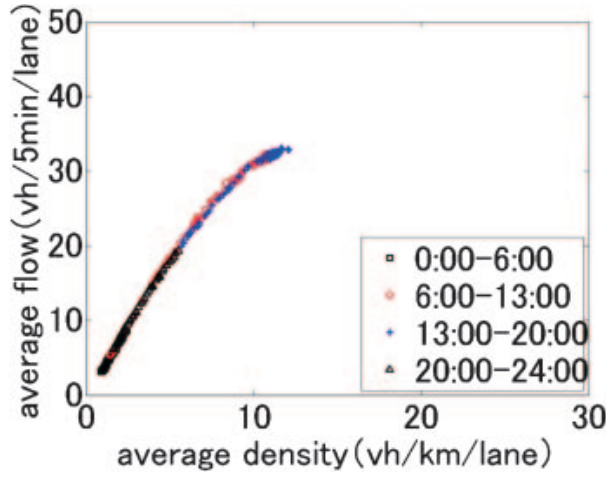

(a) MFD

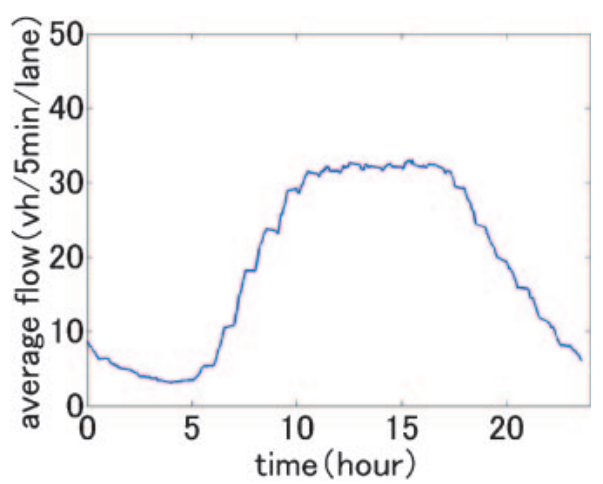

(b) Average flow

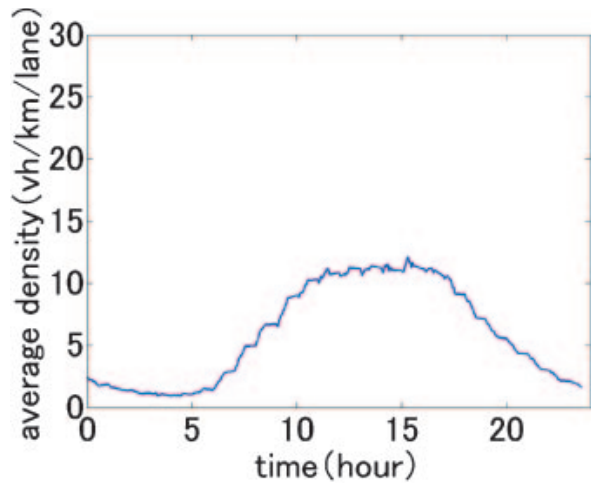

(c) Average density

Fig. 9. MFD, time-series of the average flow, and average density of 3/24/2013 (Sunday, sunny).

So far, we have classified the MFDs into two groups called the weekday and weekend based on the heuristic method. The validity of this classification can be confirmed with a more objective statistical technique. Figure 10 shows that the classification of MFDs in 10/2012 with the hierarchical clustering method (see Appendix I for details).

This result is consistent with the results from the heuristic method classification. The results also show that the MFD shape for Monday (or the first weekday after a holiday) is distinctly different from those of the other four weekdays. This corresponds to the two pattern types seen in Figs. 4(b) and 6(b) of the evening MFDs in Section 3.3. The difference between Monday and the other four weekdays (Tuesday-Friday) MFDs can be distinguished by each of the following features:

- Weekday (Tuesday-Friday) MFDs have double loops (corresponding to the morning and evening) during a single day (see Fig. 3(a)).

- Monday (or the first weekday after a holiday) MFDs have a single loop only in the morning (see Fig. 3(b)).

We can explain why the Monday (or first weekday after a holiday) MFD loops do not form in the evening in conjunction with the time-series patterns of the average flow and average density. Figure 7(a) shows that the timeseries pattern of the average flow of Monday is similar to other weekdays (a large single peak exists) in the morning; however, its peak level is low in the evening. In addition, the peak level of the average density on Monday evening is

"We choose one month for which no data records are missing. 


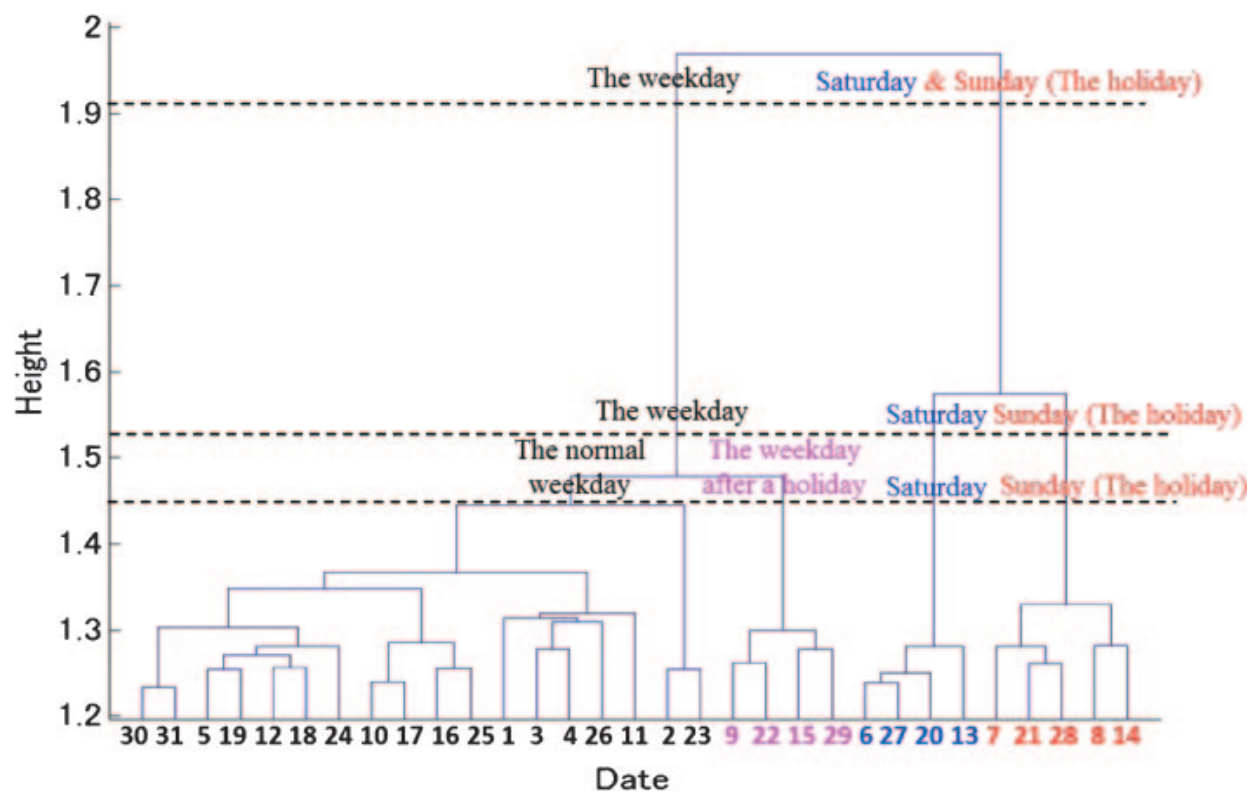

Fig. 10. Hierarchical clustering classification results.

small (see Fig. 7(b)) and not sustained. In other words, the demand level is low during Monday evening peak hours (because user behavioral characteristics change depending on the day of the week). Therefore, the congestion spread level is low at that time and loops do not form in Monday evening MFDs.

\subsection{Weather effect}

In addition to the effect on the MFD from the demand conditions described in Section 3.4, the road network services supply conditions (e.g., the vehicle driving environment may change because of rain or snow) may also affect the MFD shapes and features. Indeed, by observing the MFDs for bad weather days throughout the entire year, we find that the MFDs for bad weather days share the following common features:

- The loop is different from the good weather weekend loop.

- The lower curve of the loop has a wider density range than the loop for sunny weekends (see Figs. 11(a) and 12(a)).

The features of the bad weather day MFDs can also be explained in conjunction with the time-series patterns of average density and average flow, as previously noted with the weekend MFDs in Section 3.4. We analyze the relationship between loop formation and time-series patterns of the average flow and average density (see Figs. 11(b) and 11(c)) based on an example of a snow weekend MFD in more detail. The average flow on this day (see Fig. 11(b)) increases with slope $S_{3}^{f}$ during 10:40-16:10, then after 20:00 decreases swiftly with slope $S_{4}^{f}$. On the other hand, the average density (see Fig. 11(c)) gradually increases with slope $S_{3}^{d}$ during 13:00-16:20. This increase process corresponds to the state transition on the upper curve with $S_{3}^{f} / S_{3}^{d}$ of the MFD. The average density then maintains high values during 16:20-16:50 and begins to decrease with slope $S_{4}^{d}$ at 16:50, which is later than 16:10, when the average flow begins to decrease. This decrease corresponds to the state transition on the lower curve with $S_{4}^{f} / S_{4}^{d}$ of the MFD. The decreasing slope $S_{4}^{f} / S_{4}^{d}$ of the MFD is larger than the increasing slope $S_{3}^{f} / S_{3}^{d}$ of the MFD. The results show that the state changes in a clockwise direction along the upper curve when increasing and to the lower curve (hysteresis loop) when decreasing.

The reason why this loop is larger than the loop on a good weather day (for example, a sunny Saturday) is that the decreasing slope $S_{4}^{d}$ of the average density is less steep than slope $S_{2}^{d}$ on the sunny Saturday (see Fig. 8(c)). This implies that the time required for congestion elimination increases because snow worsens the vehicle driving environment conditions (network performance drops). In addition, we can explain the loop (see Fig. 12(a)) that is formed on rainy weekend using the same mechanism.

\subsection{Area setting effect}

Up to this point, we have analyzed the MFDs for Sendai metropolitan area road networks (see Fig. 2). However, a change of the area setting (the detector set) may also affect the MFDs features. As the first step, we should verify the effect by setting four areas with different sizes (see Fig. 13 and Table 1):

- Area 1: the minimum area corresponding to the Sendai CBD only

- Area 2: twice the number of links in area 1, which includes the nearby area 1 main roads

- Area 3: twice the number of links in area 2, which is the area enclosed by the ring freeways of the Sendai metropolitan area 


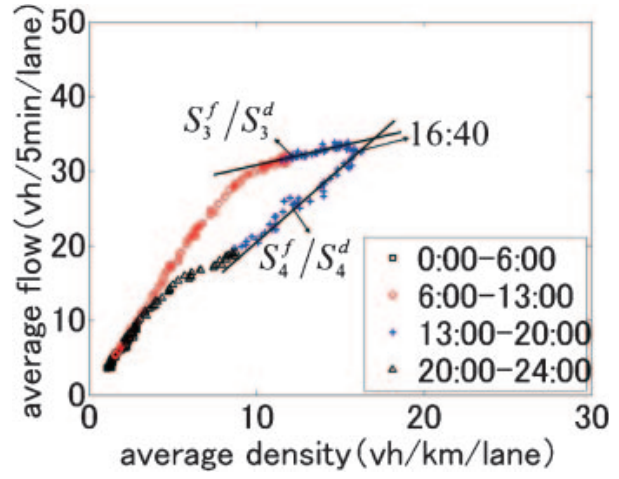

(a) MFD

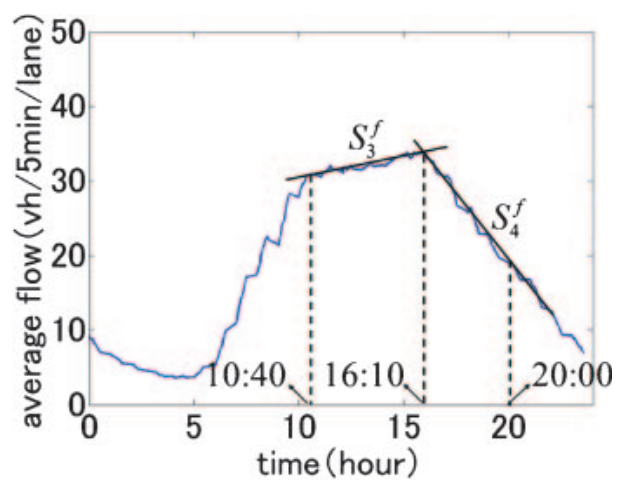

(b) Average flow

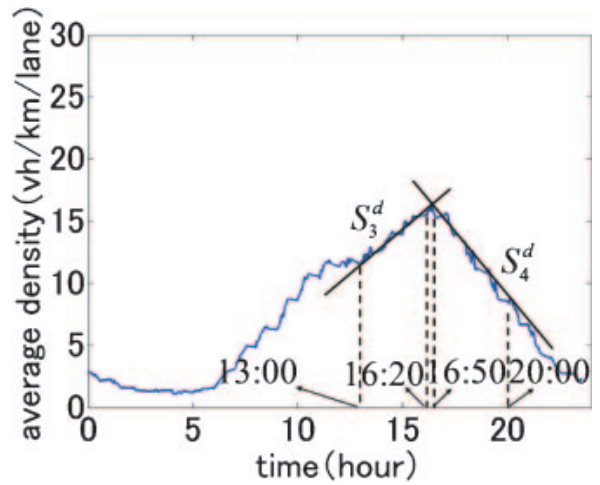

(c) Average density

Fig. 11. MFD, time-series of the average flow, and average density of 12/23/2012 (Sunday, snow).

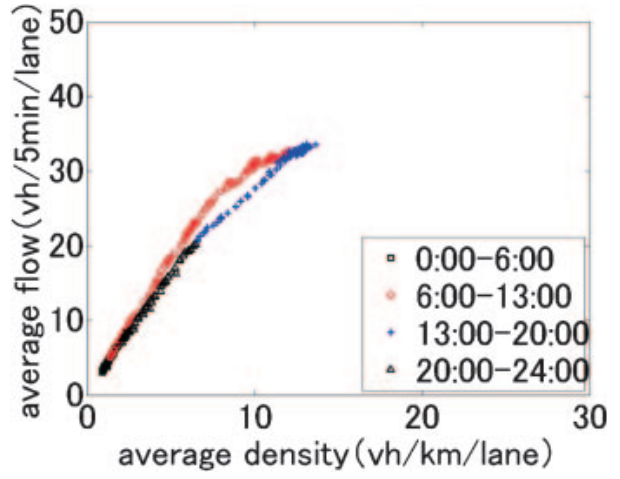

(a) MFD

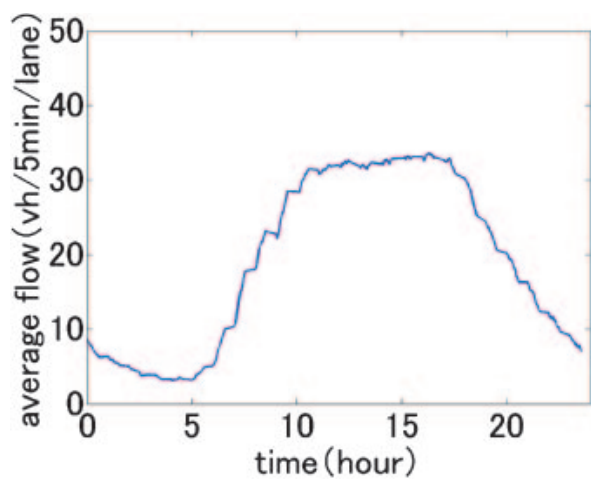

(b) Average flow

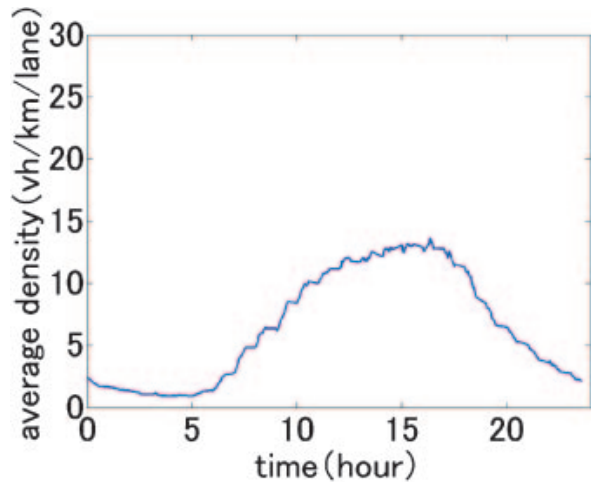

(c) Average density

Fig. 12. MFD, time-series of the average flow, and average density of 3/10/2013 (Sunday, rain).

- Area 0: twice the number of links in area 3, which is the Sendai metropolitan area (see Fig. 2, analysis area from Sections 3.2 to 3.5 )

We calculated the MFDs throughout the entire year for each area setting defined above, and show typical examples in Fig. 14. We report the results by comparing these MFDs:

- the range of average density that could be taken decreases if we set a larger area,

- when the area becomes larger, the "variation of the MFD" (the range of average flow observed for each average density, and the range of average density observed for each average flow) decreases.

We believe that the first feature is caused by the decrease of an "averaging effect," that is, the effect on the ratio of local congestion to average density in the whole area with the increase in area size. In this study, the number of suburban roads that are not too congested (only low ranges of traffic density are observed) increases as the area increases, and the proportion of congested roads (detectors) to the total number of roads (detectors) decreases ${ }^{\S}$.

We can explain the second feature using the time-series pattern of the average flow and average density in different areas. When the changes in time-series average flow and average density are not gradual, the "variation of the MFD" is large (see Figs. 14(a) and 15). However, when the changes are gradual, the "variation of the MFD" becomes smaller

${ }^{\S}$ However, the lower average density caused by the "averaging effect" becomes weak in area 3 because it contains Route 4 , one of the busiest main roads in the Sendai metropolitan area. 
Table 1. Number of detectors and the surface area of different areas.

\begin{tabular}{lcccc}
\hline & Area 1 $\left(I_{1}\right)$ & Area 2 $\left(I_{2}\right)$ & Area 3 $\left(I_{3}\right)$ & Area 0 $\left(I_{0}\right)$ \\
\hline Area $\left(\mathrm{km}^{2}\right)$ & 3.2 & 22.7 & 117.8 & \\
Number of links with detectors & 100 & 203 & 411 & 878 \\
Number of detectors & 234 & 419 & 836 & 1756 \\
\hline
\end{tabular}

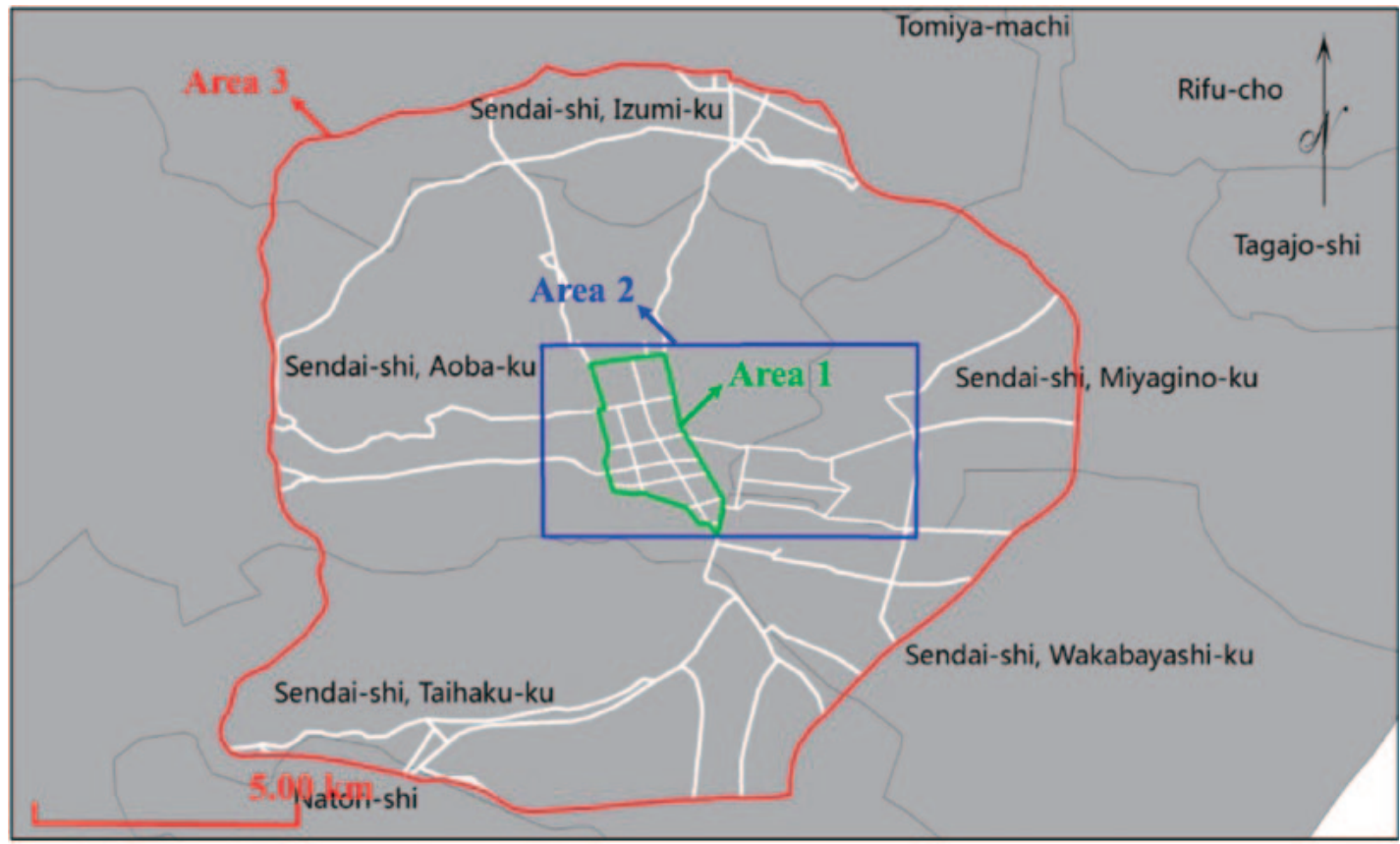

Fig. 13. Locations of areas 1-3.

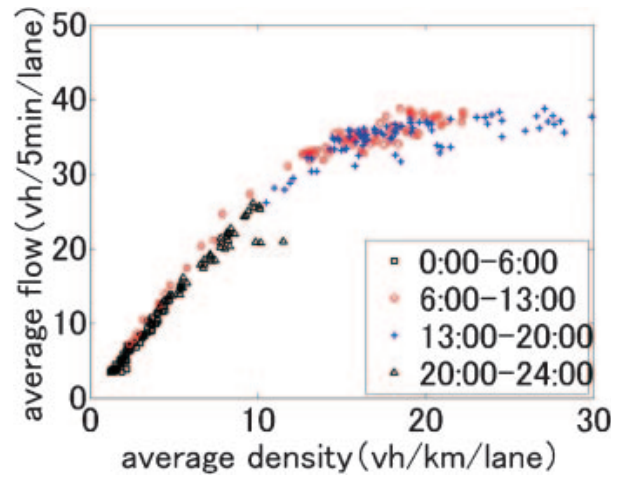

(a) Area 1

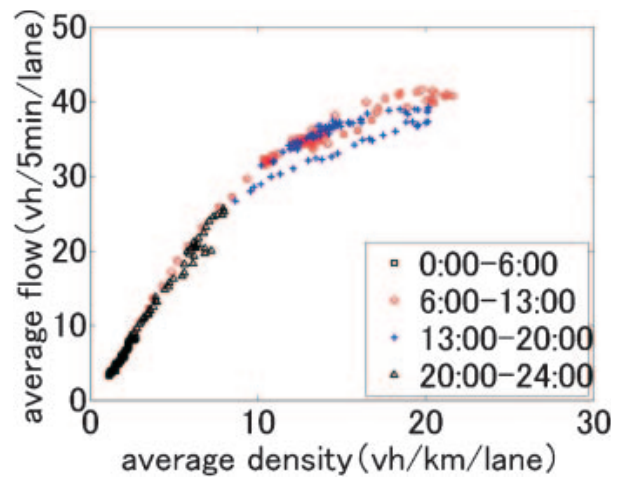

(c) Area 3

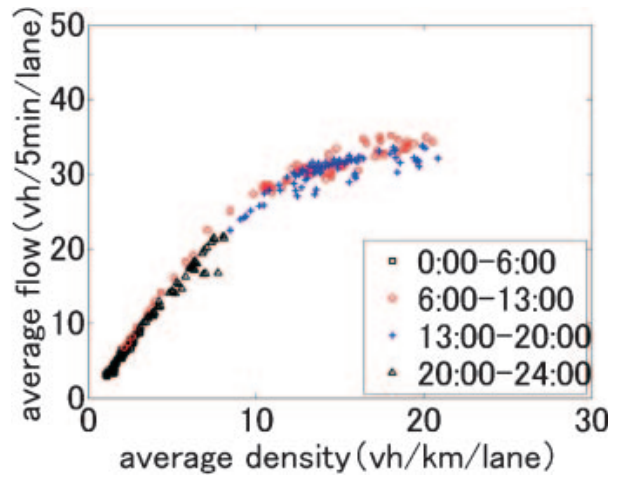

(b) Area 2

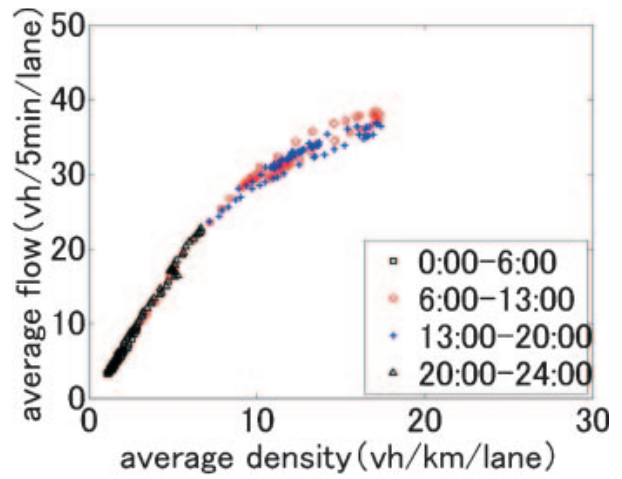

(d) Area 0

Fig. 14. MFDs in different areas for 11/7/2012 (Weekday, sunny). 


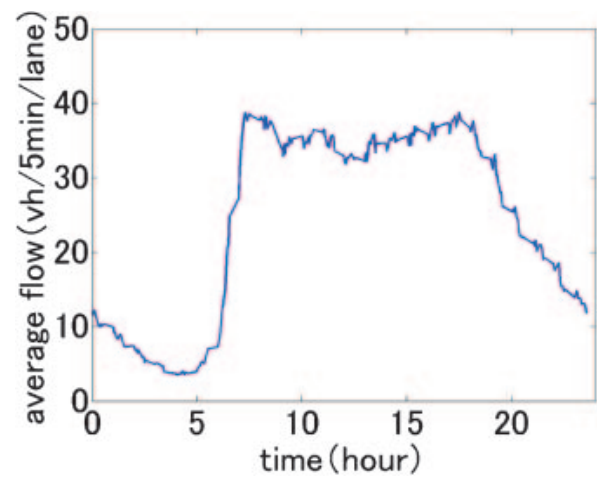

(a) Average flow

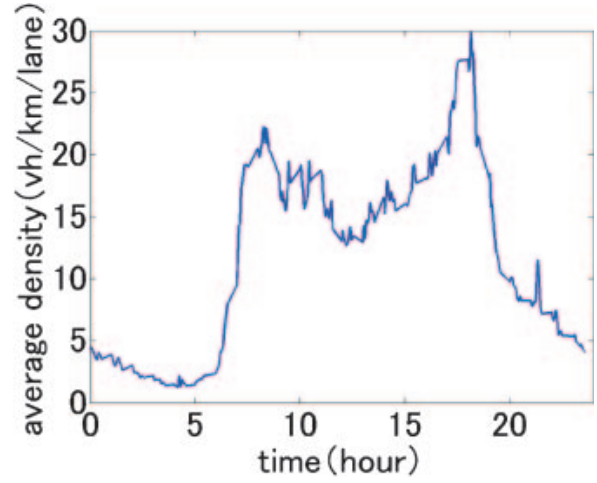

(b) Average density

Fig. 15. Time-series of the average flow and average density of 11/7/2012 in area 1 .

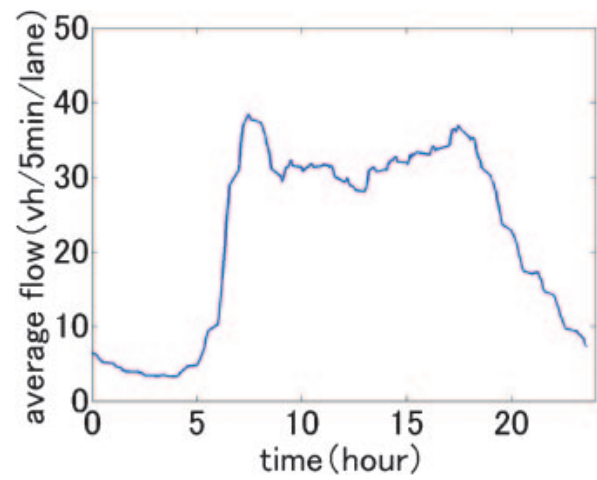

(a) Average flow

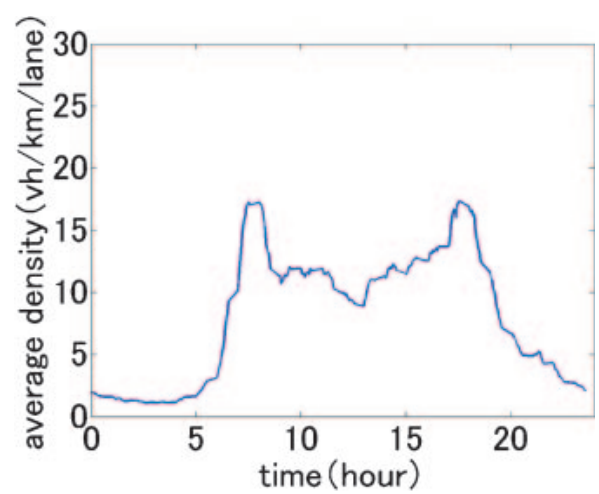

(b) Average density

Fig. 16. Time-series of the average flow and average density of $11 / 7 / 2012$ in area 0 .

(see Figs. 14(d) and 16). Therefore, we believe that the "variation of the MFD" can be explained in conjunction with the changes in the time-series patterns of average density and average flow.

In order to express the changes of the time-series of the average flow (average density), we divide the average flow (average density) into two terms: a drift term $f_{q}(t)\left(f_{k}(t)\right)$ and a random term $\sigma_{q}(t)\left(\sigma_{k}(t)\right)$, where the random term $\sigma_{q}(t)$ $\left(\sigma_{k}(t)\right)$ presents the variation of the average flow and average density for each time period $t$. In this study, we only discuss the random term change with respect to the number of detectors. When the number of detectors increases, the effect of a single detector on the average value becomes smaller, therefore $\sigma_{q}(t)\left(\sigma_{k}(t)\right)$ decreases. The "variation of the MFD" also decreases on the whole. Indeed, in this study, the number of detectors increases with the increasing area size. Therefore, in Figs. 15 and 16, we can see that the changes of average flow and average density in area 0 are smaller than those in area 1. Furthermore, the "variation of the MFD" also decreases with increasing numbers of detectors.

From the above analysis, we can see that a trade-off exists between the area size and MFD variation. We plan to quantify this trade-off and develop a method for optimal area (or detector) setting in future research.

\section{Conclusion}

We explored the MFD features for the road networks of Sendai, Japan. In order to achieve this, we used the observed data of 5-minute periods throughout one year (5/1/2012-4/30/2013) to draw the MFD for Sendai road networks. We found the following:

- a hysteresis loop always forms in the MFDs for good weather weekdays, and their formation times correspond to the morning and evening peak hours;

- a large single loop forms during one day in a Saturday MFD;

- the loop does not form in a Sunday MFD;

- a loop that is larger than that of the typical MFD forms in the MFD for a bad weather day;

- the variation of the MFD increases as the analysis area for drawing the MFD decreases.

We have shown that the MFD features can be understood in conjunction with the increases and decreases in average flow and average density time-series pattern. Specifically, our results have shown that the formation of a hysteresis 
loop in an MFD corresponds to the principle that the decrease time of the average density is later (maintains for a period of time near the peak value) than the average flow's (decreases immediately) after peak achievement. We believe that such (time-series) behavior of the average flow and average density corresponds to the spatial spreading of traffic congestion. The verification and explication of such mechanisms are very important future research topics in this area.

\section{Acknowledgments}

This work was partially supported by the Japan Society for the Promotion of Science, Grant-in-Aid for Young Scientists (B) 26820207. We would like to thank Japan Road Traffic Information Center (JARTIC) for providing us with the detector data. Furthermore, we also thank two anonymous referees for their valuable comments and suggestions.

\section{REFERENCES}

[1] Buisson, C., and Ladier, C., "Exploring the impact of homogeneity of traffic measurements on the existence of macroscopic fundamental diagrams," Transportation Research Record: Journal of the Transportation Research Board, 2124(12): 127-136 (2009).

[2] Daganzo, C. F., "Urban gridlock: Macroscopic modeling and mitigation approaches," Transportation Research Part B, 41(1): 49-62 (2007).

[3] Geroliminis, N., and Daganzo, C., "Existence of urban-scale macroscopic fundamental diagrams: Some experimental findings," Transportation Research Part B, 42(9): 759-770 (2008).

[4] Geroliminis, N., and Sun, J., "Hysteresis phenomena of a macroscopic fundamental diagram in freeway networks," Transportation Research Part A, 45(9): 966-979 (2011).

[5] Knoop, V. L., and Hoogendoorn, S. P., "Empirics of a generalized macroscopic fundamental diagram for urban freeways," Transportation Research Record: Journal of the Transportation Research Board, 2391(13): 133-141 (2013).

[6] Saberi, M., and Mahmassani, H. S., "Exploring properties of networkwide flow-density relations in a freeway network," Transportation Research Record: Journal of the Transportation Research Board, 2315(16): 153-163 (2012).

[7] Saberi, M., and Mahmassani, H. S., "Hysteresis and capacity drop phenomena freeway networks: Empirical characterization and interpretation," Transportation Research Record: Journal of the Transportation Research Board, 2391(5): 44-55 (2013).

[8] Ward, J. H., Jr., "Hierarchical grouping to optimize an objective function," Journal of the American Statistical Association, 58: 236-244 (1963).

[9] Yoshii, T., Yonezawa, Y., and Kitamura, R., "Evaluation of an area metering control method using the macroscopic fundamental diagram," The 12th World Conference on Transport Research, 1-12 (2010). 


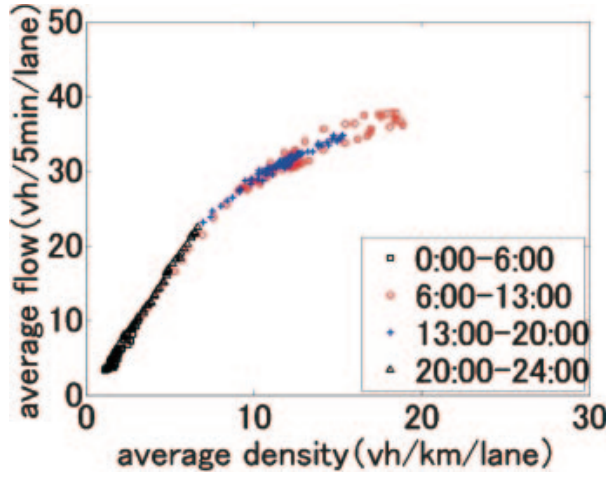

(a) Plots of the 24-hour MFD

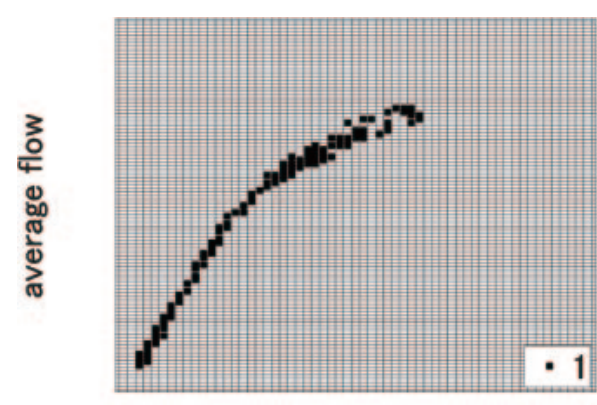

average density

(b) The meshed MFD

Fig. 17. Meshed MFD example.

\section{Appendix I: Clustering Classification Method}

This appendix presents the hierarchical clustering method algorithm adopted in Section 3.4. We need to explain two points about this algorithm that differentiate it from a normal hierarchical clustering method: (1) we use the Ward Method (see Ward (1963)) to define the distance between two clusters and (2) we define the distance matrix (degree of the difference between two MFDs) by using 0-1 vector, which is based on the meshed MFD. The outline of this algorithm can be summarized as follows:

Step 0: $n=N$ ( $N$ is the number of MFDs.)

Step 1: Create the $n \times n$ distance matrix $\mathbf{D}$.

Step 2: Summarize two clusters to one cluster by Ward Method.

Step 3: $n=n-1$

Step 4: If $n=1$, then terminate, otherwise $n>1$ and return to Step 2.

We only describe the definition of the distance matrix $\mathbf{D}$ in detail. First, we generate the mesh for the horizontal and vertical axes (Fig. 17).

Second, we represent the shape of the MFD for each day by a $0-1$ vector $\mathbf{M}$ as follows:

$$
M_{i j}= \begin{cases}1 & \text { if } P_{i j}>0 \\ 0 & \text { if } P_{i j}=0\end{cases}
$$

where $M_{i j}$ is calculated using the number of plots $P_{i j}$ in the mesh. Furthermore, we calculate the effective mesh number of day $a$ and day $b, N_{a}$ and $N_{b}$, respectively, as well as the common effective mesh number $N_{a \cap b}$, as follows:

$$
\left\{\begin{array}{c}
N_{a}=\sum_{i=1}^{I} \sum_{j=1}^{J} M_{i j}^{a} \\
N_{b}=\sum_{i=1}^{I} \sum_{j=1}^{J} M_{i j}^{b} \\
N_{a \cap b}=\sum_{i=1}^{I} \sum_{j=1}^{J} M_{i j}^{a} M_{i j}^{b}
\end{array}\right.
$$

where $I$ is the total number of meshes on the vertical axis and $J$ is the total number of meshes on the horizontal axis. Finally, distance $D_{a b}$ between day $a$ and day $b$ can be defined as follows:

$$
D_{a b}=\frac{N_{a}+N_{b}-2 N_{a \cap b}}{N_{a}+N_{b}-N_{a \cap b}}
$$




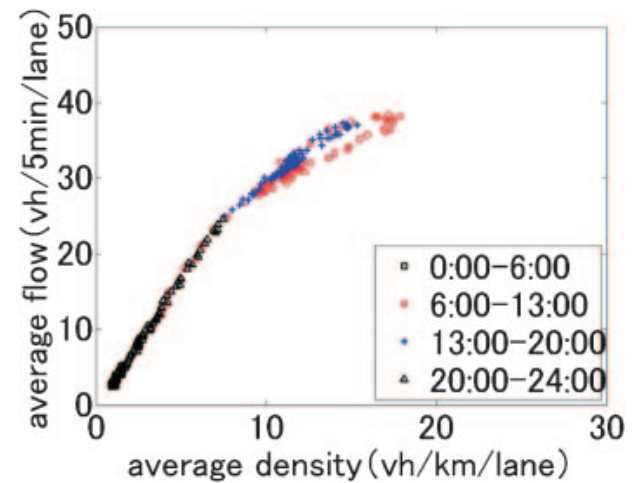

(a) 6/2012/4 (Monday, sunny)

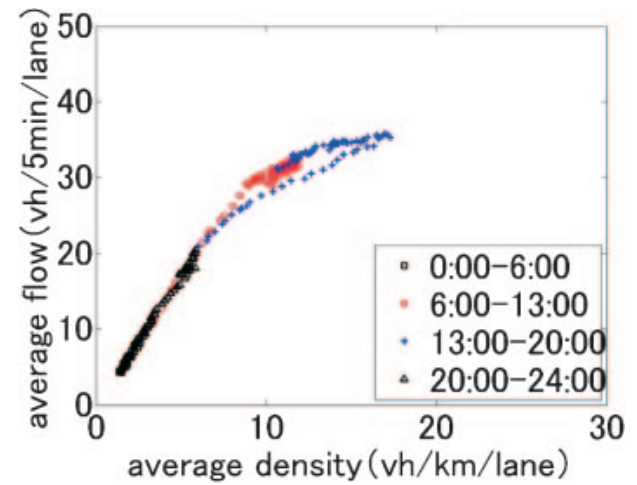

(c) 10/2012/6 (Saturday, sunny)

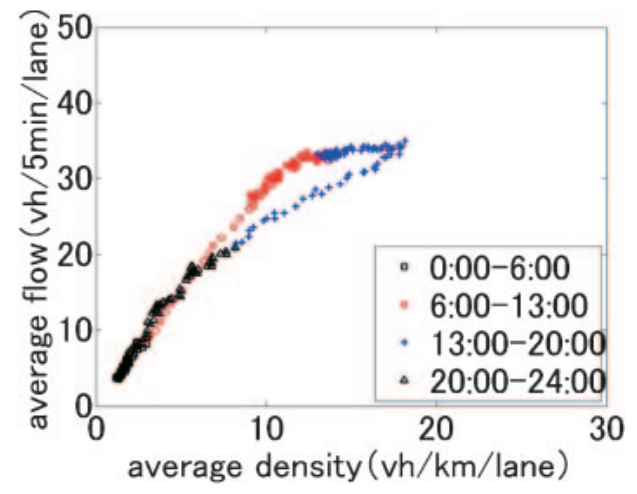

(e) $12 / 2012 / 8$ (Saturday, snow)

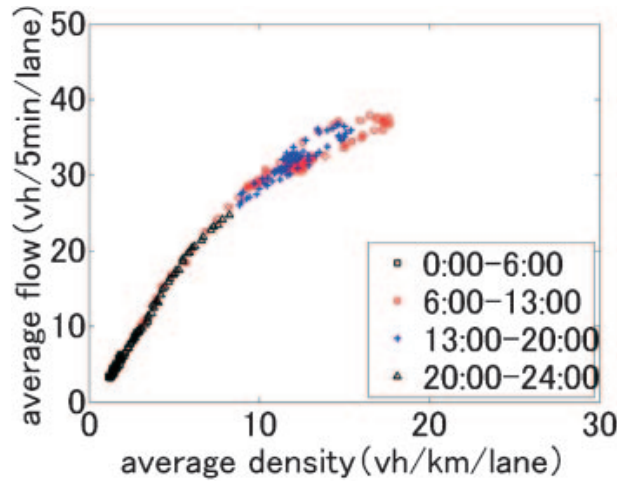

(b) 5/2012/10 (Thursday, sunny)

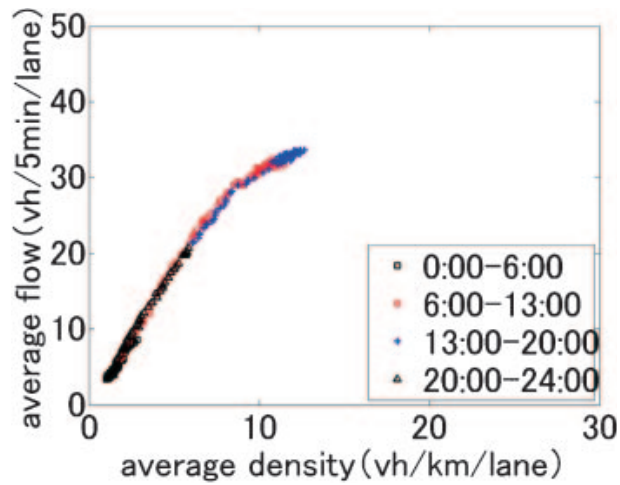

(d) $7 / 2012 / 8$ (Sunday, sunny)

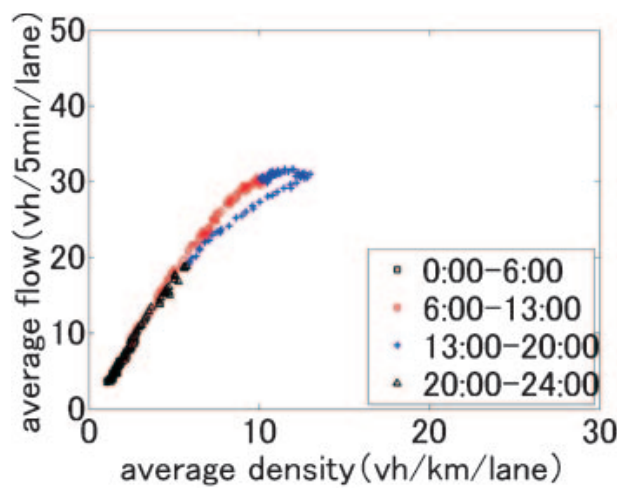

(f) $9 / 2012 / 2$ (Sunday, rain)

Fig. 18. MFDs of other dates for area 0 under various conditions.

\section{Appendix II: MFDs of Other Dates}

This appendix presents some MFDs of other dates with different conditions throughout the entire year (5/1/20124/30/2012). Specially, Figs. 18(a) and 18(b) are MFDs on weekdays with good weather (standard condition), Figs. 18(c) and 18(d) are MFDs on sunny weekends, Figs. 18(e) and 18(f) are MFDs on bad weather days. 